\title{
The Eigen Theory of Electromagnetic Waves in Complex Media
}

\author{
Shaohua Guo \\ Zhejiang University of Science and Technology \\ P. R. China
}

\section{Introduction}

Since J. C. Maxwell presented the electromagnetic field equations in 1873, the existence of electromagnetic waves has been verified in various medium (Kong, 1986; Monk, 2003). But except for Helmholtz's equation of electromagnetic waves in isotropic media, the laws of propagation of electromagnetic waves in anisotropic media are not clear to us yet. For example, how many electromagnetic waves are there in anisotropic media? How fast can these electromagnetic waves propagate? Where are propagation direction and polarization direction of the electromagnetic waves? What are the space patterns of these waves? Although many research works were made in trying to deduce the equations of electromagnetic waves in anisotropic media based on the Maxwell's equation (Yakhno, 2005, 2006; Cohen, 2002; Haba, 2004), the explicit equations of electromagnetic waves in anisotropic media could not be obtained because the dielectric permittivity matrix and magnetic permeability matrix were all included in these equations, so that only local behaviour of electromagnetic waves, for example, in a certain plane or along a certain direction, can be studied.

On the other hand, it is a natural fact that electric and magnetic fields interact with each other in classical electromagnetics. Therefore, even if most of material studies deal with the properties due to dielectric polarisation, magneitc materials are also capable of producing quite interesting electro-magnetic effects (Lindellm et al., 1994). From the bi-anisotropic point of view, magnetic materials can be treated as a subclass of magnetoelectric materials. The linear constitutive relations linking the electric and magnetic fields to the electric and magnetic displacements contain four dyadics, three of which have direct magnetic contents. The magnetoelectric coupling has both theoretical and practical significance in solid state physics and materials science. Though first predicted by Pierre Curie, magnetoeletric coupling was originally through to be forbidden because it violates time-reversal symmetry, until Laudau and Lifshitz (Laudau \& Lifshitz, 1960) pointed out that time reversal is not a symmetry operation in some magnetic crystal. Based on this argument, Dzyaloshinskii (Dzyaloshinskii, 1960) predicted that magnetoelectric effect should occur in antiferromagnetic crystal $\mathrm{Cr}_{2} \mathrm{O}_{3}$, which was verified experimentally by Astrov (Astrov, 1960). Since then the magnetoelectric coupling has been observed in single-phase materials where simultaneous electric and magnetic ordering coexists, and in two-phase composites where the participating phase are pizoelectric and piezomagnetic (Bracke \& Van Vliet,1981; Van Run et al., 1974). Agyei and Birman (Agyei \& Birman, 1990) carried out a detailed 
analysis of the linear magnetoelectric effect, which showed that the effect should occur not only in some magnetic but also in some electric crystals. Pradhan (Pradhan, 1993) showed that an electric charge placed in a magnetoelectric medium becomes a source of induced magnetic field with non-zero divergence of volume integral. Magnetoelectric effect in twophase composites has been analyzed by Harshe et al. ( Harshe et al., 1993), Nan (Nan, 1994) and Benveniste (Benveniste, 1995). Broadband transducers based on magnetoelectric effect have also been developed (Bracke \& Van Vliet, 1981). Although the development mentioned above, no great progress in the theories of electromagnetic waves in bi-anisotropic media because of the difficulties in deal with the bi-coupling in electric field and magnetic one of the Maxell's equation and the bi-anisotropic constitutive equation by classical electromagnetic theory.

Recently there is a growing interest modeling and analysis of Maxwell's equations (Lee \& Madsen, 1990; Monk, 1992; Jin et al., 1999). However, most work is restricted to simple medium such as air in the free space. On the other hand, we notice that lossy and dispersive media are ubiquitous, for example human tissue, water, soil, snow, ice, plasma, optical fibers and radar-absorbing materials. Hence the study of how electromagnetic wave interacts with dispersive media becomes very important. Some concrete applications include geophysical probing and subsurface studied of the moon and other planets (Bui et al., 1991), High power and ultra-wide-band radar systems, in which it is necessary to model ultra-wide-band electromagnetic pulse propagation through plasmas (Dvorak \& Dudley, 1995), ground penetrating radar detection of buried objects in soil media (liu \& Fan, 1999). The Debye medium plays an important role in electromagnetic wave interactions with biological and water-based substances (Gandhi \& Furse, 1997). Until 1990, some paper on modeling of wave propagation in dispersive media started making their appearance in computational electromagnetics community. However, the published papers on modeling of dispersive media are exclusively restricted to the finite-difference time-domain methods and the finite element methods (Li \& Chen, 2006; Lu et al., 2004). To our best knowledge, there exist only few works in the literature, which studied the theoretical model for the Maxwell's equation in the complex anisotropic dispersive media, and no explicit equations of electromagnetic waves in anisotropic dispersive media can be obtained due to the limitations of classical electromagnetic theory.

Chiral materials have been recently an interesting subject. In a chiral medium, an electric or magnetic excitation will produce simultaneously both electric and magnetic polarizations. On the other hand, the chiral medium is an object that cannot be brought into congruence with its mirror image by translation and rotation. Chirality is common in a variety of naturally occurring and man-made objects. From an operation point of view, chirality is introduced into the classical Maxwell equations by the Drude-Born-Fedorov relative constitutive relations in which the electric and magnetic fields are coupled via a new materials parameter (Lakhtakia, 1994; Lindell et al., 1994), the chirality parameter. These constitutive relations are chosen because they are symmetric under time reversality and duality transformations. In a homogeneous isotropic chiral medium the electromagnetic fields are composed of left-circularly polarized (LCP) and right- circularly polarized (RCP) components (Jaggard et al., 1979; Athanasiadis \& Giotopoulos, 2003), which have different wave numbers and independent directions of propagation. Whenever an electromagnetic wave (LCP, RCP or a linear combination of them) is incident upon a chiral scatterer, then the scattered field is composed of both LCP and RCP components and therefore both LCP and RCP far-field patterns are derived. Hence, in the vector problem we need to specify two 
directions of propagation and two polarizations. In recent years, chiral materials have been increasingly studied and there is a growing literature covering both their applications and the theoretical investigation of their properties. It will be noticed that the works dealing with wave phenomena in chiral materials have been mainly concerned with the study of timeharmonic waves which lead to frequency domain studies (Lakhtakia et al., 1989; Athanasiadis et al., 2003).

In this chapter, the idea of standard spaces is used to deal with the Maxwell's electromagnetic equation (Guo, 2009, 2009, 2010, 2010, 2010). By this method, the classical Maxwell's equation under the geometric presentation can be transformed into the eigen Maxwell's equation under the physical presentation. The former is in the form of vector and the latter is in the form of scalar. Through inducing the modal constitutive equations of complex media, such as anisotropic media, bi-anisotropic media, lossy media, dissipative media, and chiral media, a set of modal equations of electromagnetic waves for all of those media are obtained, each of which shows the existence of electromagnetic sub-waves, meanwhile its propagation velocity, propagation direction, polarization direction and space pattern can be completely determined by the modal equations.This chapter will make introductions of the eigen theory to reader in details. Several novel theoretical results were discussed in the different parts of this chapter.

\section{Standard spaces of electromagnetic media}

In anisotropic electromagnetic media, the dielectric permittivity and magnetic permeability are tensors instead of scalars. The constitutive relations are expressed as follows

$$
D=\varepsilon \cdot E, \quad B=\mu \cdot H
$$

Rewriting Eq.(1) in form of scalar, we have

$$
D_{i}=\varepsilon_{i j} E_{j}, \quad B_{i}=\mu_{i j} H_{j}
$$

where the dielectric permittivity matrix $\varepsilon$ and the magnetic permeability matrix $\boldsymbol{\mu}$ are usually symmetric ones, and the elements of the matrixes have a close relationship with the selection of reference coordinate. Suppose that if the reference coordinates is selected along principal axis of electrically or magnetically anisotropic media, the elements at non-diagonal of these matrixes turn to be zero. Therefore, equations (1) and (2) are called the constitutive equations of electromagnetic media under the geometric presentation. Now we intend to get rid of effects of geometric coordinate on the constitutive equations, and establish a set of coordinate-independent constitutive equations of electromagnetic media under physical presentation. For this purpose, we solve the following problems of eigen-value of matrixes.

$$
(\varepsilon-\lambda I) \phi=0, \quad(\mu-\gamma I) \varphi=0
$$

where $\lambda_{i}(i=1,2,3)$ and $\gamma_{i}(i=1,2,3)$ are respectively eigen dielectric permittivity and eigen magnetic permeability, which are constants of coordinate-independent. $\phi_{i}(i=1,2,3)$ and $\boldsymbol{\varphi}_{i}(i=1,2,3)$ are respectively eigen electric vector and eigen magnetic vector, which show the electrically principal direction and magnetically principal direction of anisotropic media, and are all coordinate-dependent. We call these vectors as standard spaces. Thus, the matrix 
of dielectric permittivity and magnetic permeability can be spectrally decomposed as follows

$$
\boldsymbol{\varepsilon}=\boldsymbol{\Phi} \Lambda \boldsymbol{\Phi}^{\mathrm{T}}, \quad \boldsymbol{\mu}=\boldsymbol{\Psi} \Pi \boldsymbol{\Psi}^{\mathrm{T}}
$$

where $\boldsymbol{\Lambda}=\operatorname{diag}\left[\lambda_{1}, \lambda_{2}, \lambda_{3}\right]$ and $\boldsymbol{\Pi}=\operatorname{diag}\left[\gamma_{1}, \gamma_{2}, \gamma_{3}\right]$ are the matrix of eigen dielectric permittivity and eigen magnetic permeability, respectively. $\boldsymbol{\Phi}=\left\{\boldsymbol{\phi}_{1}, \boldsymbol{\phi}_{2}, \boldsymbol{\phi}_{3}\right\}$ and $\boldsymbol{\Psi}=\left\{\boldsymbol{\varphi}_{1}, \boldsymbol{\varphi}_{2}, \boldsymbol{\varphi}_{3}\right\}$ are respectively the modal matrix of electric media and magnetic media, which are both orthogonal and positive definite matrixes, and satisfy $\boldsymbol{\Phi}^{\mathrm{T}} \boldsymbol{\Phi}=\boldsymbol{I}, \boldsymbol{\Psi}^{\mathrm{T}} \boldsymbol{\Psi}=\boldsymbol{I}$.

Projecting the electromagnetic physical qualities of the geometric presentation, such as the electric field intensity vector $\boldsymbol{E}$, magnetic field intensity vector $\boldsymbol{H}$, magnetic flux density vector $\boldsymbol{B}$ and electric displacement vector $\boldsymbol{D}$ into the standard spaces of the physical presentation, we get

$$
\begin{array}{ll}
\boldsymbol{D}^{*}=\boldsymbol{\Phi}^{\mathrm{T}} \cdot \boldsymbol{D}, & \boldsymbol{E}^{*}=\boldsymbol{\Phi}^{\mathrm{T}} \cdot \boldsymbol{E} \\
\boldsymbol{B}^{*}=\boldsymbol{\Psi}^{\mathrm{T}} \cdot \boldsymbol{B}, \quad \boldsymbol{H}^{*}=\boldsymbol{\Psi}^{\mathrm{T}} \cdot \boldsymbol{H}
\end{array}
$$

Rewriting Eqs.(5) and (6) in the form of scalar, we have

$$
\begin{array}{cccc}
D_{i}^{*}=\boldsymbol{\phi}_{i}^{\mathrm{T}} \cdot \boldsymbol{D} & i=1,2,3, E_{i}^{*}=\boldsymbol{\phi}_{i}^{\mathrm{T}} \cdot \boldsymbol{E} & i=1,2,3 \\
B_{i}^{*}=\boldsymbol{\varphi}_{i}^{\mathrm{T}} \cdot \boldsymbol{B} & i=1,2,3 \quad, H_{i}^{*}=\boldsymbol{\varphi}_{i}^{\mathrm{T}} \cdot \boldsymbol{H} & i=1,2,3
\end{array}
$$

These are the electromagnetic physical qualities under the physical presentation.

Substituting Eq. (4) into Eq. (1) respectively, and using Eqs.(5) and (6) yield

$$
\begin{array}{ll}
D_{i}^{*}=\lambda_{i} E_{i}^{*} & i=1,2,3 \\
B_{i}^{*}=\gamma_{i} H_{i}^{*} & i=1,2,3
\end{array}
$$

The above equations are just the modal constitutive equations in the form of scalar.

\section{Eigen expression of Maxwell's equation}

The classical Maxwell's equations in passive region can be written as

$$
\boldsymbol{\nabla} \times \boldsymbol{H}=\nabla_{t} \boldsymbol{D}, \quad \boldsymbol{\nabla} \times \boldsymbol{E}=-\nabla_{t} \boldsymbol{B}
$$

Now we rewrite the equations in the form of matrix as follows

$$
\left[\begin{array}{ccr}
0 & -\partial_{z} & \partial_{y} \\
\partial_{z} & 0 & -\partial_{x} \\
-\partial_{y} & \partial_{x} & 0
\end{array}\right]\left\{\begin{array}{l}
H_{1} \\
H_{2} \\
H_{3}
\end{array}\right\}=\nabla_{t}\left\{\begin{array}{l}
D_{1} \\
D_{2} \\
D_{3}
\end{array}\right\}
$$

or

$$
[\Delta]\{H\}=\nabla_{t}\{D\}
$$




$$
\left[\begin{array}{ccc}
0 & -\partial_{z} & \partial_{y} \\
\partial_{z} & 0 & -\partial_{x} \\
-\partial_{y} & \partial_{x} & 0
\end{array}\right]\left\{\begin{array}{l}
E_{1} \\
E_{2} \\
E_{3}
\end{array}\right\}=-\nabla_{t}\left\{\begin{array}{l}
B_{1} \\
B_{2} \\
B_{3}
\end{array}\right\}
$$

or

$$
[\Delta]\{E\}=-\nabla_{t}\{B\}
$$

where $[\Delta]$ is defined as the matrix of electric and magnetic operators.

Substituting Eq. (1) into Eqs. (13) and (15) respectively, we have

$$
\begin{gathered}
{[\Delta]\{H\}=\nabla_{t}[\varepsilon]\{E\}} \\
{[\Delta]\{E\}=-\nabla_{t}[\mu]\{H\}}
\end{gathered}
$$

Substituting Eq. (16) into (17) or Eq. (17) into (16), yield

$$
\begin{gathered}
{[\square]\{H\}=-\nabla_{t}^{2}[\mu][\varepsilon]\{H\}} \\
{[\square]\{E\}=-\nabla_{t}^{2}[\mu][\varepsilon]\{E\}}
\end{gathered}
$$

where $[\square]=[\Delta][\Delta]$ is defined as the matrix of electromagnetic operators as follows

$$
[\square]=\left[\begin{array}{ccc}
-\left(\partial_{z}^{2}+\partial_{y}^{2}\right) & \partial_{x y}^{2} & \partial_{x z}^{2} \\
\partial_{y x}^{2} & -\left(\partial_{x}^{2}+\partial_{z}^{2}\right) & \partial_{y z}^{2} \\
\partial_{z x}^{2} & \partial_{z y}^{2} & -\left(\partial_{x}^{2}+\partial_{y}^{2}\right)
\end{array}\right]
$$

In another way, substituting Eqs. (5) and (6) into Eqs. (13) and (15), respectively, we have

$$
\begin{aligned}
& {[\Delta][\Phi]\left\{E^{*}\right\}=-\nabla_{t}[\Psi]\left\{B^{*}\right\}} \\
& {[\Delta][\Psi]\left\{H^{*}\right\}=\nabla_{t}[\Phi]\left\{D^{*}\right\}}
\end{aligned}
$$

Rewriting the above in indicial notation, we get

$$
\begin{aligned}
& \left\{\Delta_{i}^{*}\right\} E_{i}^{*}=-\nabla_{t}\left\{\varphi_{i}\right\} B_{i}^{*} \quad i=1,2,3 \\
& \left\{\Delta_{i}^{*}\right\} H_{i}^{*}=\nabla_{t}\left\{\phi_{i}\right\} D_{i}^{*} \quad i=1,2,3
\end{aligned}
$$

where, $\Delta_{i}^{*}$ is the electromagnetic intensity operator, and $i$ th row of $\left[\Delta^{*}\right]=[\Delta][\Phi]$.

\section{Electromagnetic waves in anisotropic media}

\subsection{Electrically anisotropic media}

In anisotropic dielectrics, the dielectric permittivity is a tensor, while the magnetic permeability is a scalar. So Eqs. (18) and (19) can be written as follows 


$$
\begin{gathered}
{[\square]\{H\}=-\nabla_{t}^{2} \mu_{0}[\varepsilon]\{H\}} \\
{[\square]\{E\}=-\nabla_{t}^{2} \mu_{0}[\varepsilon]\{E\}}
\end{gathered}
$$

Substituting Eqs. (4) - (6) into Eqs. (25) and (26), we have

$$
\begin{aligned}
& {\left[\square^{*}\right]\left\{H^{*}\right\}=-\nabla_{t}^{2} \mu_{0}[\Lambda]\left\{H^{*}\right\}} \\
& {\left[\square^{*}\right]\left\{E^{*}\right\}=-\nabla_{t}^{2} \mu_{0}[\Lambda]\left\{E^{*}\right\}}
\end{aligned}
$$

where $\left[\square^{*}\right]=[\Phi]^{\mathrm{T}}[\square][\Phi]$ is defined as the eigen matrix of electromagnetic operators under the standard spaces. We can note from Appendix A that it is a diagonal matrix. Thus Eqs. (27) and (28) can be uncoupled in the form of scalar

$$
\begin{array}{cc}
\square_{i}^{*} H_{i}^{*}+\mu_{0} \lambda_{i} \nabla_{t}^{2} H_{i}^{*}=0 & i=1,2,3 \\
\square_{i}^{*} E_{i}^{*}+\mu_{0} \lambda_{i} \nabla_{t}^{2} E_{i}^{*}=0 & i=1,2,3
\end{array}
$$

Eqs.(29) and (30) are the modal equations of electromagnetic waves in anisotropic dielectrics.

\subsection{Magnetically anisotropic media}

In anisotropic magnetics, the magnetic permeability is a tensor, while the dielectric permittivity is a scalar. So Eqs. (18) and (19) can be written as follows

$$
\begin{gathered}
{[\square]\{H\}=-\nabla_{t}^{2} \varepsilon_{0}[\mu]\{H\}} \\
{[\square]\{E\}=-\nabla_{t}^{2} \varepsilon_{0}[\mu]\{E\}}
\end{gathered}
$$

Substituting Eqs. (4) - (6) into Eqs. (31) and (32), we have

$$
\begin{aligned}
& {\left[\square^{*}\right]\left\{H^{*}\right\}=-\nabla_{t}^{2} \varepsilon_{0}[\Pi]\left\{H^{*}\right\}} \\
& {\left[\square^{*}\right]\left\{E^{*}\right\}=-\nabla_{t}^{2} \varepsilon_{0}[\Pi]\left\{E^{*}\right\}}
\end{aligned}
$$

where $\left[\square^{*}\right]=[\Psi]^{\mathrm{T}}[\square][\Psi]$ is defined as the eigen matrix of electromagnetic operators under the standard spaces. We can also note from Appendix A that it is a diagonal matrix. Thus Eqs. (33) and (34) can be uncoupled in the form of scalar

$$
\begin{array}{rl}
\square_{i}^{*} H_{i}^{*}+\varepsilon_{0} \gamma_{i} \nabla_{t}^{2} H_{i}^{*}=0 & i=1,2,3 \\
\square_{i}^{*} E_{i}^{*}+\varepsilon_{0} \gamma_{i} \nabla_{t}^{2} E_{i}^{*}=0 & i=1,2,3
\end{array}
$$

Eqs.(35) and (36) are the modal equations of electromagnetic waves in anisotropic magnetics. 


\section{Electromagnetic waves in bi-anisotropic media}

\section{$5.1 \mathrm{Bi}$-anisotropic constitutive equations}

The constitutive equations of bi-anisotropic media are the following (Lindellm \& Sihvola, 1994; Laudau \& Lifshitz, 1960)

$$
\begin{aligned}
& D=\varepsilon \cdot E+\xi \cdot H \\
& B=\xi \cdot E+\mu \cdot H
\end{aligned}
$$

where $\boldsymbol{\xi}$ is the matrix of magneto-electric parameter, and a symmetric one.

Substituting Eqs. (5) and (6) into Eqs. (37) and (38), respectively, and multiplying them with the transpose of modal matrix in the left, we have

$$
\begin{gathered}
\boldsymbol{\Phi}^{\mathrm{T}} \boldsymbol{D}=\boldsymbol{\Phi}^{\mathrm{T}} \boldsymbol{\varepsilon} \boldsymbol{\Phi} \boldsymbol{E}^{*}+\boldsymbol{\Phi}^{\mathrm{T}} \boldsymbol{\xi} \boldsymbol{\Psi} \boldsymbol{H}^{*} \\
\boldsymbol{\Psi}^{\mathrm{T}} \boldsymbol{B}=\boldsymbol{\Psi}^{\mathrm{T}} \boldsymbol{\xi} \boldsymbol{\Phi} \boldsymbol{E}^{*}+\boldsymbol{\Psi}^{\mathrm{T}} \boldsymbol{\mu} \boldsymbol{\Psi} \boldsymbol{H}^{*}
\end{gathered}
$$

Let $\boldsymbol{G}=\boldsymbol{\Phi}^{\mathrm{T}} \boldsymbol{\xi} \boldsymbol{\Psi}=\boldsymbol{\Psi}^{\mathrm{T}} \boldsymbol{\xi} \boldsymbol{\Phi}$, that is a coupled magneto-electric matrix, and using Eq. (4), we have

$$
\begin{aligned}
& \boldsymbol{D}^{*}=\boldsymbol{\Lambda} \boldsymbol{E}^{*}+\boldsymbol{G} \boldsymbol{H}^{*} \\
& \boldsymbol{B}^{*}=\boldsymbol{G} \boldsymbol{E}^{*}+\boldsymbol{\Pi} \boldsymbol{H}^{*}
\end{aligned}
$$

Rewriting the above in indicial notation, we get

$$
\begin{array}{lll}
D_{i}^{*}=\lambda_{i} E_{i}^{*}+g_{i j} H_{j}^{*} & i=1,2,3 & j=1,2,3 \\
B_{i}^{*}=\gamma_{i} H_{i}^{*}+g_{i j} E_{j}^{*} & i=1,2,3 & j=1,2,3
\end{array}
$$

Eqs. (43) and (44) are just the modal constitutive equations for bi-anisotropic media.

\subsection{Eigen equations of electromagnetic waves in bi-anisotropic media} Substituting Eqs. (43) and (44) into Eqs. (23) and (24), respectively, we have

$$
\begin{gathered}
\left\{\Delta_{i}^{*}\right\} E_{i}^{*}=-\nabla_{t}\left\{\varphi_{i}\right\}\left(\gamma_{i} H_{i}^{*}+g_{i j} E_{j}^{*}\right) \\
\left\{\Delta_{i}^{*}\right\} H_{i}^{*}=\nabla_{t}\left\{\phi_{i}\right\}\left(\lambda_{i} E_{i}^{*}+g_{i j} H_{j}^{*}\right)
\end{gathered}
$$

From them, we can get

$$
\begin{gathered}
\left(\left\{\Delta_{i}^{*}\right\}-\nabla_{t}\left\{\phi_{i}\right\} g_{i j} \delta_{i j}\right)^{T}\left(\left\{\Delta_{i}^{*}\right\}+\nabla_{t}\left\{\varphi_{i}\right\} g_{i j} \delta_{i j}\right) E_{i}^{*}=-\nabla_{t}^{2}\left\{\varphi_{i}\right\}\left\{\phi_{i}\right\}^{T} \lambda_{i} \gamma_{i} E_{i}^{*} \\
\left(\left\{\Delta_{i}^{*}\right\}+\nabla_{t}\left\{\varphi_{i}\right\} g_{i j} \delta_{i j}\right)^{T}\left(\left\{\Delta_{i}^{*}\right\}-\nabla_{t}\left\{\phi_{i}\right\} g_{i j} \delta_{i j}\right) H_{i}^{*}=-\nabla_{t}^{2}\left\{\phi_{i}\right\}\left\{\varphi_{i}\right\}^{T} \gamma_{i} \lambda_{i} H_{i}^{*}
\end{gathered}
$$

The above can also be written as the standard form of waves 


$$
\begin{array}{cc}
\square_{i}^{*} E_{i}^{*}+\nabla_{t}\left\{\Delta_{i}^{*}\right\}^{T} \cdot(\{\varphi\}-\{\phi\}) g_{i i} E_{i}^{*}+\nabla_{t}^{2}\{\phi\}^{T} \cdot\{\varphi\}\left(\lambda_{i} \gamma_{i}-g_{i i}^{2}\right) E_{i}^{*}=0 & i=1,2,3 \\
\square_{i}^{*} H_{i}^{*}+\nabla_{t}\left\{\Delta_{i}^{*}\right\}^{T} \cdot(\{\varphi\}-\{\phi\}) g_{i i} H_{i}^{*}+\nabla_{t}^{2}\{\phi\}^{T} \cdot\{\varphi\}\left(\lambda_{i} \gamma_{i}-g_{i i}^{2}\right) H_{i}^{*}=0 \quad i=1,2,3
\end{array}
$$

where, $\square_{i}^{*}=\left\{\Delta_{i}^{*}\right\}^{T} \cdot\left\{\Delta_{i}^{*}\right\}$ is the electromagnetic operator. Eqs.(49) and (50) are just equations of electric field and magnetic field for bi-anisotropic media.

\subsection{Applications}

\subsubsection{Bi-isotropic media}

The constitutive equations of bi-isotropic media are the following

$$
\begin{aligned}
\boldsymbol{D} & =\left[\begin{array}{lll}
\varepsilon & 0 & 0 \\
0 & \varepsilon & 0 \\
0 & 0 & \varepsilon
\end{array}\right] \cdot \boldsymbol{E}+\left[\begin{array}{ccc}
\boldsymbol{\xi} & 0 & 0 \\
0 & \xi & 0 \\
0 & 0 & \xi
\end{array}\right] \cdot \boldsymbol{H} \\
\boldsymbol{B} & =\left[\begin{array}{lll}
\xi & 0 & 0 \\
0 & \xi & 0 \\
0 & 0 & \xi
\end{array}\right] \cdot \boldsymbol{E}+\left[\begin{array}{ccc}
\mu & 0 & 0 \\
0 & \mu & 0 \\
0 & 0 & \mu
\end{array}\right] \cdot \boldsymbol{H}
\end{aligned}
$$

The eigen values and eigen vectors of those matrix are the following

$$
\begin{gathered}
\boldsymbol{\Lambda}=\operatorname{diag}[\varepsilon, \varepsilon, \varepsilon], \quad \boldsymbol{\Pi}=\operatorname{diag}[\mu, \mu, \mu] \\
\boldsymbol{\Phi}=\boldsymbol{\Psi}=\left[\begin{array}{lll}
1 & 0 & 0 \\
0 & 1 & 0 \\
0 & 0 & 1
\end{array}\right]
\end{gathered}
$$

We can see from the above equations that there is only one eigen-space in isotropic medium, which is a triple-degenerate one, and the space structure is the following

$$
\begin{gathered}
\boldsymbol{W}=W_{1}^{(3)}\left[\phi_{1}, \phi_{2}, \phi_{3}\right] \\
\boldsymbol{\phi}^{*}=\boldsymbol{\varphi}_{1}^{*}=\frac{\sqrt{3}}{3}[1,1,1]^{T}
\end{gathered}
$$

Then the eigen-qualities and eigen-operators of bi-isotropic medium are respectively shown as belows

$$
\begin{gathered}
E_{1}^{*}=\phi_{1}^{* \mathrm{~T}} \cdot \boldsymbol{E}=\frac{\sqrt{3}}{3}\left(E_{1}+E_{2}+E_{3}\right) \\
\square_{1}^{*}=-\left(\partial_{x}^{2}+\partial_{y}^{2}+\partial_{z}^{2}\right), g_{11}=\xi
\end{gathered}
$$

So, the equation of electromagnetic wave in bi-isotropic medium becomes 


$$
\left(\partial_{x}^{2}+\partial_{y}^{2}+\partial_{z}^{2}\right) E_{1}^{*}=\left(\mu \varepsilon-\xi^{2}\right) \partial_{t}^{2} E_{1}^{*}
$$

the velocity of electromagnetic wave is

$$
c^{(1)}=\frac{1}{\sqrt{\mu \varepsilon-\xi^{2}}}
$$

\subsubsection{Dzyaloshinskii's bi-anisotropic media}

Dzyaloshinskii's constitutive equations of bi-anisotropic media are the following

$$
\begin{aligned}
\boldsymbol{D} & =\left[\begin{array}{lll}
\varepsilon & 0 & 0 \\
0 & \varepsilon & 0 \\
0 & 0 & \varepsilon_{z}
\end{array}\right] \cdot \boldsymbol{E}+\left[\begin{array}{ccc}
\xi & 0 & 0 \\
0 & \xi & 0 \\
0 & 0 & \xi_{z}
\end{array}\right] \cdot \boldsymbol{H} \\
\boldsymbol{B} & =\left[\begin{array}{lll}
\xi & 0 & 0 \\
0 & \xi & 0 \\
0 & 0 & \xi_{z}
\end{array}\right] \cdot \boldsymbol{E}+\left[\begin{array}{ccc}
\mu & 0 & 0 \\
0 & \mu & 0 \\
0 & 0 & \mu_{z}
\end{array}\right] \cdot \boldsymbol{H}
\end{aligned}
$$

The eigen values and eigen vectors of those matrix are the following

$$
\begin{gathered}
\boldsymbol{\Lambda}=\operatorname{diag}\left[\varepsilon, \varepsilon, \varepsilon_{z}\right], \quad \boldsymbol{\Pi}=\operatorname{diag}\left[\mu, \mu, \mu_{z}\right] \\
\boldsymbol{\Phi}=\boldsymbol{\Psi}=\left[\begin{array}{lll}
1 & 0 & 0 \\
0 & 1 & 0 \\
0 & 0 & 1
\end{array}\right]
\end{gathered}
$$

We can see from the above equations that there are two eigen-spaces in Dzyaloshinskii's bianisotropic medium, in which one is a binary-degenerate one, the space structure is the following

$$
\boldsymbol{W}=W_{1}^{(2)}\left[\phi_{1}, \phi_{2}\right] \oplus W_{2}^{1}\left[\phi_{3}\right]
$$

Then the eigen-qualities and eigen-operators of Dzyaloshinskii's bi-anisotropic medium are respectively shown as belows

$$
\begin{gathered}
E_{2}^{*}=\boldsymbol{\phi}_{2}^{\mathrm{T}} \cdot \boldsymbol{E}=E_{3}, \quad\left|E_{1}^{*}\right|=\sqrt{\left(\boldsymbol{E}-\boldsymbol{\phi}_{2}^{\mathrm{T}} E_{2}^{*}\right)^{\mathrm{T}}\left(\boldsymbol{E}-\boldsymbol{\phi}_{2}^{\mathrm{T}} E_{2}^{*}\right)}=\sqrt{E_{1}^{2}+E_{2}^{2}} \\
\square_{1}^{*}=-\left(\partial_{x}^{2}+\partial_{y}^{2}+2 \partial_{z}^{2}-2 \partial_{x y}^{2}\right), \quad \square_{2}^{*}=-\left(\partial_{x}^{2}+\partial_{y}^{2}\right), \quad g_{11}=\xi, \quad g_{22}=\xi_{z}
\end{gathered}
$$

So, the equations of electromagnetic wave in Dzyaloshinskii's bi-anisotropic medium become

$$
\begin{gathered}
\left(\partial_{x}^{2}+\partial_{y}^{2}+2 \partial_{z}^{2}-2 \partial_{x y}^{2}\right) \sqrt{E_{1}^{2}+E_{2}^{2}}=\left(\mu \varepsilon-\xi^{2}\right) \partial_{t}^{2} \sqrt{E_{1}^{2}+E_{2}^{2}} \\
\left(\partial_{x}^{2}+\partial_{y}^{2}\right) E_{3}=\left(\mu_{z} \varepsilon_{z}-\xi_{z}^{2}\right) \partial_{t}^{2} E_{3}
\end{gathered}
$$


the velocities of electromagnetic wave are

$$
\begin{gathered}
c^{(1)}=\frac{1}{\sqrt{\mu \varepsilon-\xi^{2}}} \\
c^{(2)}=\frac{1}{\sqrt{\mu_{z} \varepsilon_{z}-\xi_{z}^{2}}}
\end{gathered}
$$

It is seen both from bi-isotropic media and Dzyaloshinskii's bi-anisotropic medium that the electromagnetic waves in bi-anisotropic medium will go faster duo to the bi-coupling between electric field and magnetic one.

\section{Electromagnetic waves in lossy media}

\subsection{The constitutive equation of lossy media}

The constitutive equation of lossy media is the following

$$
\boldsymbol{D}=\boldsymbol{\varepsilon} \cdot \boldsymbol{E}+\int_{t} \sigma \cdot \frac{d \boldsymbol{E}}{d \tau} d \tau
$$

It is equivalent to the following differential constitutive equation

$$
\dot{\boldsymbol{D}}=\boldsymbol{\varepsilon} \cdot \dot{\boldsymbol{E}}+\sigma \cdot \boldsymbol{E}
$$

Let

$$
\boldsymbol{D}^{\mathrm{e}}=\boldsymbol{\varepsilon} \cdot \boldsymbol{E}, \quad \dot{\boldsymbol{D}}^{\mathrm{d}}=\sigma \cdot \boldsymbol{E}
$$

Eq.(73) can be written as

$$
\dot{\boldsymbol{D}}=\dot{\boldsymbol{D}}^{\mathrm{e}}+\dot{\boldsymbol{D}}^{\mathrm{d}}
$$

or

$$
\nabla_{t}\{D\}=\left([\varepsilon] \nabla_{t}+[\sigma]\right)\{E\}
$$

Using Eq.(5), the above becomes

$$
\nabla_{t}\left\{D^{*}\right\}=\left([\Phi]^{T}[\varepsilon][\Phi] \nabla_{t}+[\Phi]^{T}[\sigma][\Phi]\right)\left\{E^{*}\right\}
$$

According to Appendix B and Eq.(77), we have

$$
\nabla_{t}\left\{D^{*}\right\}=\left([\Lambda] \nabla_{t}+[\Gamma]\right)\left\{E^{*}\right\}
$$

Rewriting the above in indicial notation, we get

$$
\nabla_{t} D_{i}^{*}=\left(\lambda_{i} \nabla_{t}+\eta_{i}\right) E_{i}^{*}
$$

Eq.(79) is just the modal constitutive equations for lossy media. 


\subsection{Eigen equations of electromagnetic waves in lossy media}

Substituting Eqs. (10) and (79) into Eqs. (23) and (24), respectively, we have

$$
\begin{gathered}
\left\{\Delta_{i}^{*}\right\} E_{i}^{*}=-\nabla_{t}\left\{\varphi_{i}\right\} \gamma_{i} H_{i}^{*} \quad i=1,2,3 \\
\left\{\Delta_{i}^{*}\right\} H_{i}^{*}=\left\{\phi_{i}\right\}\left(\lambda_{i} \nabla_{t}+\eta_{i}\right) E_{i}^{*} \quad i=1,2,3
\end{gathered}
$$

From them, we can get

$$
\begin{array}{cc}
\square_{i}^{*} E_{i}^{*}+\nabla_{t t} \xi_{i} \gamma_{i} \lambda_{i} E_{i}^{*}+\nabla_{t} \xi_{i} \gamma_{i} \eta_{i} E_{i}^{*}=0 & i=1,2,3 \\
\square_{i}^{*} H_{i}^{*}+\nabla_{t t} \xi_{i} \gamma_{i} \lambda_{i} H_{i}^{*}+\nabla_{t} \xi_{i} \gamma_{i} \eta_{i} H_{i}^{*}=0 & i=1,2,3
\end{array}
$$

where $\xi_{i}=\left\{\phi_{i}^{*}\right\}^{T} \cdot\left\{\varphi_{i}^{*}\right\}$. Eqs.(82) and (83) are just equations of electric field and magnetic field for bi-anisotropic media.

\subsection{Applications}

In this section, we discuss the propagation laws of electromagnetic waves in an isotropic lossy medium. The material tensors in Eqs.(1) and (72) are represented by the following matrices

$$
\boldsymbol{\varepsilon}=\left[\begin{array}{lll}
\varepsilon_{11} & 0 & 0 \\
0 & \varepsilon_{11} & 0 \\
0 & 0 & \varepsilon_{11}
\end{array}\right], \boldsymbol{\mu}=\left[\begin{array}{lll}
\mu_{11} & 0 & 0 \\
0 & \mu_{11} & 0 \\
0 & 0 & \mu_{11}
\end{array}\right], \boldsymbol{\sigma}=\left[\begin{array}{lll}
\sigma_{11} & 0 & 0 \\
0 & \sigma_{11} & 0 \\
0 & 0 & \sigma_{11}
\end{array}\right]
$$

The eigen values and eigen vectors of those matrix are the following

$$
\begin{gathered}
\boldsymbol{\Lambda}=\operatorname{diag}\left[\varepsilon_{11}, \varepsilon_{11}, \varepsilon_{11}\right], \boldsymbol{\Pi}=\operatorname{diag}\left[\mu_{11}, \mu_{11}, \mu_{11}\right], \boldsymbol{\Gamma}=\operatorname{diag}\left[\sigma_{11}, \sigma_{11}, \sigma_{11}\right] \\
\boldsymbol{\Phi}=\boldsymbol{\Psi}=\boldsymbol{\Theta}=\left[\begin{array}{lll}
1 & 0 & 0 \\
0 & 1 & 0 \\
0 & 0 & 1
\end{array}\right]
\end{gathered}
$$

We can see from the above equations that there is only one eigen-space in an isotropic lossy medium, which is a triple-degenerate one, and the space structure is the following.

$$
\boldsymbol{W}_{\text {mag }}=W_{1}^{(3)}\left[\boldsymbol{\phi}_{1}, \boldsymbol{\phi}_{2}, \boldsymbol{\phi}_{3}\right], \boldsymbol{W}_{\text {ele }}=W_{1}^{(3)}\left[\boldsymbol{\varphi}_{1}, \boldsymbol{\varphi}_{2}, \boldsymbol{\varphi}_{3}\right]
$$

where, $\phi_{1}^{*}=\frac{\sqrt{3}}{3}\{1,1,1\}^{T}, \varphi_{1}^{*}=\frac{\sqrt{3}}{3}\{1,1,1\}^{T}, \xi_{1}=1$.

Then the eigen-qualities and eigen-operators of an isotropic lossy media are respectively shown as follows

$$
E_{1}^{*}=\frac{\sqrt{3}}{3}\left(E_{1}+E_{2}+E_{3}\right)
$$




$$
\begin{aligned}
& H_{1}^{*}=\frac{\sqrt{3}}{3}\left(H_{1}+H_{2}+H_{3}\right) \\
& \square_{1}^{*}=\frac{1}{3}\left[-\left(\partial_{x}^{2}+\partial_{y}^{2}+\partial_{z}^{2}\right)\right]
\end{aligned}
$$

So, the equation of electromagnetic wave in lossy media becomes

$$
\left(\partial_{x}^{2}+\partial_{y}^{2}+\partial_{z}^{2}\right) E_{1}^{*}=\frac{1}{c^{2}} \partial_{t}^{2} E_{1}^{*}+\frac{1}{\tau^{2}} \partial_{t} E_{1}^{*}
$$

Rewriting it in the component form, we have

$$
\begin{aligned}
& \left(\partial_{x}^{2}+\partial_{y}^{2}+\partial_{z}^{2}\right) E_{1}=\frac{1}{c^{2}} \partial_{t}^{2} E_{1}+\frac{1}{\tau^{2}} \partial_{t} E_{1} \\
& \left(\partial_{x}^{2}+\partial_{y}^{2}+\partial_{z}^{2}\right) E_{2}=\frac{1}{c^{2}} \partial_{t}^{2} E_{2}+\frac{1}{\tau^{2}} \partial_{t} E_{2} \\
& \left(\partial_{x}^{2}+\partial_{y}^{2}+\partial_{z}^{2}\right) E_{3}=\frac{1}{c^{2}} \partial_{t}^{2} E_{3}+\frac{1}{\tau^{2}} \partial_{t} E_{3}
\end{aligned}
$$

where, $c$ is the velocity of electromagnetic wave, $\tau$ is the lossy coefficient of electromagnetic wave

$$
c=\frac{1}{\sqrt{\mu_{11} \varepsilon_{11}}}, \tau=\frac{1}{\sqrt{\mu_{11} \sigma_{11}}}
$$

Now, we discuss the the propagation laws of a plane electromagnetic wave in $x$-axis. In this time, Eq. (92) becomes

$$
\frac{\partial}{\partial x^{2}} E_{1}=\frac{1}{c^{2}} \partial_{t}^{2} E_{1}+\frac{1}{\tau^{2}} \partial_{t} E_{1}
$$

Let the solution of Eq. (96) is as follows

$$
E_{1}=A \exp [i(k x-\omega t)]
$$

Substituting the above into Eq. (96), we have

$$
k^{2}=\frac{\omega^{2}}{c^{2}}+i \frac{\omega}{\tau^{2}}
$$

From Eq.(96), we can get

$$
\bar{k}=k_{1}+i k_{2}
$$


where

$$
k_{1}=\frac{\omega}{c}\left[\frac{1+\left(1+\frac{c^{4}}{\omega^{2} \tau^{4}}\right)^{\frac{1}{2}}}{2}\right]^{\frac{1}{2}}, k_{2}=\frac{\omega}{c}\left[\frac{-1+\left(1+\frac{c^{4}}{\omega^{2} \tau^{4}}\right)^{\frac{1}{2}}}{2}\right]^{\frac{1}{2}}
$$

Then, the solutions of electromagnetic waves are the following

$$
E_{1}=A e^{-k_{2} x} \cdot e^{i\left(k_{1} x-\omega t\right)}=\bar{A} \cdot e^{i\left(k_{1} x-\omega t\right)}
$$

It is an attenuated sub-waves.

\section{Electromagnetic waves in dispersive media}

\subsection{The constitutive equation of dispersive media}

The general constitutive equations of dispersive media are the following

$$
\begin{gathered}
D=\boldsymbol{\varepsilon} \cdot \boldsymbol{E}+\boldsymbol{\varepsilon}_{1} \cdot \dot{\boldsymbol{E}}+\boldsymbol{\varepsilon}_{2} \cdot \ddot{\boldsymbol{E}}+\cdots \\
\boldsymbol{B}=\boldsymbol{\mu} \cdot \boldsymbol{H}+\mu_{1} \cdot \dot{\boldsymbol{H}}+\mu_{2} \cdot \ddot{\boldsymbol{H}}+\cdots
\end{gathered}
$$

where $\boldsymbol{\varepsilon}_{i},(i=1,2, \cdots)$ and $\boldsymbol{\mu}_{i},(i=1,2, \cdots)$ are the higher order dielectric permittivity matrix and the magnetic permeability matrix respectively, and all symmtric ones.

Substituting Eqs. (5) and (6) into Eqs. (101) and (102), respectively, and multiplying them with the transpose of modal matrix in the left, we have

$$
\begin{gathered}
\boldsymbol{D}^{*}=\boldsymbol{\Phi}^{T} \boldsymbol{\varepsilon} \boldsymbol{\Phi} \boldsymbol{E}^{*}+\boldsymbol{\Phi}^{T} \boldsymbol{\varepsilon}_{1} \boldsymbol{\Phi} \dot{\boldsymbol{E}}+\boldsymbol{\Phi}^{T} \boldsymbol{\varepsilon}_{2} \boldsymbol{\Phi} \ddot{\boldsymbol{E}}+\cdots \\
\boldsymbol{B}^{*}=\boldsymbol{\Psi}^{T} \boldsymbol{\mu} \boldsymbol{\Psi} \boldsymbol{H}+\boldsymbol{\Psi}^{T} \boldsymbol{\mu}_{1} \boldsymbol{\Psi} \dot{\boldsymbol{H}}+\boldsymbol{\Psi}^{T} \boldsymbol{\mu}_{2} \boldsymbol{\Psi} \ddot{\boldsymbol{H}}+\cdots
\end{gathered}
$$

It can be proved that there exist same standard spaces for various order electric and magnetic fields in the condition close to the thermodynamic equilibrium. Then, we have

$$
\begin{gathered}
D_{i}^{*}=\lambda_{i} E_{i}^{*}+\lambda_{i}^{(1)} \dot{E}_{i}^{*}+\lambda_{i}^{(2)} \ddot{E}_{i}^{*}+\cdots \\
B_{i}^{*}=\gamma_{i} H_{i}^{*}+\gamma_{i}^{(1)} \dot{H}_{i}^{*}+\gamma_{i}^{(2)} \ddot{H}_{i}^{*}+\cdots
\end{gathered}
$$

Eqs. (105) and (106) are just the modal constitutive equations for the general dispersive media.

\subsection{Eigen equations of electromagnetic waves in dispersive media}

Substituting Eqs. (105) and (106) into Eqs. (23) and (24), respectively, we have

$$
\left\{\Delta_{i}^{*}\right\} E_{i}^{*}=-\nabla_{t}\left\{\varphi_{i}\right\}\left(\gamma_{i} H_{i}^{*}+\gamma_{i}^{(1)} \dot{H}_{i}^{*}+\gamma_{i}^{(2)} \ddot{H}_{i}^{*}+\cdots\right)
$$




$$
\left\{\Delta_{i}^{*}\right\} H_{i}^{*}=\nabla_{t}\left\{\phi_{i}\right\}\left(\lambda_{i} E_{i}^{*}+\lambda_{i}^{(1)} \dot{E}_{i}^{*}+\lambda_{i}^{(2)} \ddot{E}_{i}^{*}+\cdots\right)
$$

From them, we can get

$$
\begin{gathered}
\frac{1}{\xi_{i}} \square_{i}^{*} E_{i}^{*}+\gamma_{i} \lambda_{i} \nabla_{t t t} E_{i}^{*}+\left(\gamma_{i} \lambda_{i}^{(1)}+\gamma_{i}^{(1)} \lambda_{i}\right) \nabla_{t t t} E_{i}^{*}+\left(\gamma_{i} \lambda_{i}^{(2)}+\gamma_{i}^{(1)} \lambda_{i}^{(1)}+\gamma_{i}^{(2)} \lambda_{i}\right) \nabla_{t t t t} E_{i}^{*}+\cdots=0 \\
\frac{1}{\xi_{i}} \square_{i}^{*} H_{i}^{*}+\gamma_{i} \lambda_{i} \nabla_{t t} H_{i}^{*}+\left(\gamma_{i} \lambda_{i}^{(1)}+\gamma_{i}^{(1)} \lambda_{i}\right) \nabla_{t t t} H_{i}^{*}+\left(\gamma_{i} \lambda_{i}^{(2)}+\gamma_{i}^{(1)} \lambda_{i}^{(1)}+\gamma_{i}^{(2)} \lambda_{i}\right) \nabla_{t t t t} H_{i}^{*}+\cdots=0
\end{gathered}
$$

Eqs.(109) and (110) are just equations of electric field and magnetic field for general dispersive media.

\subsection{Applications}

In this section, we discuss the propagation laws of electromagnetic waves in an one-order dispersive medium. The material tensors in Eqs.(101) and (102) are represented by the following matrices

$$
\boldsymbol{\varepsilon}=\left[\begin{array}{lll}
\varepsilon_{11} & 0 & 0 \\
0 & \varepsilon_{11} & 0 \\
0 & 0 & \varepsilon_{11}
\end{array}\right], \boldsymbol{\mu}=\left[\begin{array}{lll}
\mu_{11} & 0 & 0 \\
0 & \mu_{11} & 0 \\
0 & 0 & \mu_{11}
\end{array}\right], \boldsymbol{\varepsilon}_{1}=\left[\begin{array}{lll}
\varepsilon_{11}^{\prime} & 0 & 0 \\
0 & \varepsilon_{11}^{\prime} & 0 \\
0 & 0 & \varepsilon_{11}^{\prime}
\end{array}\right], \mu_{1}=\left[\begin{array}{lll}
\mu_{11}^{\prime} & 0 & 0 \\
0 & \mu_{11}^{\prime} & 0 \\
0 & 0 & \mu_{11}^{\prime}
\end{array}\right]
$$

The eigen values and eigen vectors of those matrix are the following

$$
\begin{gathered}
\boldsymbol{\Lambda}=\operatorname{diag}\left[\varepsilon_{11}, \varepsilon_{11}, \varepsilon_{11}\right], \boldsymbol{\Lambda}_{1}=\operatorname{diag}\left[\varepsilon_{11}^{\prime}, \boldsymbol{\varepsilon}_{11}^{\prime}, \boldsymbol{\varepsilon}_{11}^{\prime}\right] \\
\boldsymbol{\Pi}=\operatorname{diag}\left[\mu_{11}, \mu_{11}, \mu_{11}\right], \boldsymbol{\Pi}_{1}=\operatorname{diag}\left[\mu_{11}^{\prime}, \mu_{11}^{\prime}, \mu_{11}^{\prime}\right] \\
\boldsymbol{\Phi}=\boldsymbol{\Psi}=\left[\begin{array}{lll}
1 & 0 & 0 \\
0 & 1 & 0 \\
0 & 0 & 1
\end{array}\right]
\end{gathered}
$$

We can see from the above equations that there is only one eigen-space in isotropic oneorder dispersive medium, which is a triple-degenerate one, and the space structure is the following

$$
\boldsymbol{W}_{\text {mag }}=W_{1}^{(3)}\left[\boldsymbol{\phi}_{1}, \boldsymbol{\phi}_{2}, \boldsymbol{\phi}_{3}\right], \boldsymbol{W}_{\text {ele }}=W_{1}^{(3)}\left[\boldsymbol{\varphi}_{1}, \boldsymbol{\varphi}_{2}, \boldsymbol{\varphi}_{3}\right]
$$

where, $\phi_{1}^{*}=\frac{\sqrt{3}}{3}\{1,1,1\}^{T}, \quad \varphi_{1}^{*}=\frac{\sqrt{3}}{3}\{1,1,1\}^{T}, \quad \xi_{1}=1$. Thus the eigen-qualities and eigenoperators of isotropic one-order dispersive medium are known as same as Eqs. (88) - (90). The equations of electromagnetic wave in one-order dispersive medium become

$$
\left(\partial_{x}^{2}+\partial_{y}^{2}+\partial_{z}^{2}\right) E_{1}^{*}=\frac{1}{c^{2}} \nabla_{t t} E_{1}^{*}+\left(\mu_{11} \varepsilon_{11}^{(1)}+\mu_{11}^{(1)} \varepsilon_{11}\right) \nabla_{t t t} E_{1}^{*}
$$




$$
\left(\partial_{x}^{2}+\partial_{y}^{2}+\partial_{z}^{2}\right) H_{1}^{*}=\frac{1}{c^{2}} \nabla_{t t} H_{1}^{*}+\left(\mu_{11} \varepsilon_{11}^{(1)}+\mu_{11}^{(1)} \varepsilon_{11}\right) \nabla_{t t t} H_{1}^{*}
$$

in which $c$ is the velocity of electromagnetic wave

$$
c=\frac{1}{\sqrt{\mu_{11} \varepsilon_{11}}}
$$

Now, we discuss the propagation laws of a plane electromagnetic wave in x-axis. In this time, Eq.(116) becomes

$$
\frac{\partial}{\partial x^{2}} E_{1}=\frac{1}{c^{2}} \nabla_{t t} E_{1}+\left(\mu_{11} \varepsilon_{11}^{(1)}+\mu_{11}^{(1)} \varepsilon_{11}\right) \nabla_{t t t} E_{1}
$$

Let

$$
E_{1}=A \exp [i(k x-\omega t)]
$$

Substituting the above into Eq.(119), we have

$$
k^{2}=\frac{\omega^{2}}{c^{2}}-i \omega^{3}\left(\mu_{11} \varepsilon_{11}^{(1)}+\mu_{11}^{(1)} \varepsilon_{11}\right)
$$

From the above, we can get

$$
\bar{k}=k_{1}+i k_{2}
$$

where

$$
k_{1}=\frac{\omega}{c}\left[\frac{1+\left(1+c^{4}\left(\mu_{11} \varepsilon_{11}^{(1)}+\mu_{11}^{(1)} \varepsilon_{11}\right)^{2} \omega^{2}\right)^{\frac{1}{2}}}{2}\right]^{\frac{1}{2}}, k_{2}=\frac{\omega}{c}\left[\frac{-1+\left(1+c^{4}\left(\mu_{11} \varepsilon_{11}^{(1)}+\mu_{11}^{(1)} \varepsilon_{11}\right)^{2} \omega^{2}\right)^{\frac{1}{2}}}{2}\right]^{\frac{1}{2}} \text {. }
$$

Then, the solutions of electromagnetic waves are

$$
E_{1}=A e^{-k_{2} x} \cdot e^{i\left(k_{1} x-\omega t\right)}=\bar{A} \cdot e^{i\left(k_{1} x-\omega t\right)}
$$

It is an attenuated sub-waves.

\section{Electromagnetic waves in chiral media}

\subsection{The constitutive equation of chiral media}

The constitutive equations of chiral media are the following

$$
\begin{aligned}
& \boldsymbol{D}=\boldsymbol{\varepsilon} \cdot \boldsymbol{E}-\boldsymbol{\chi} \cdot \nabla_{t} \boldsymbol{H} \\
& \boldsymbol{B}=\chi \cdot \nabla_{t} \boldsymbol{E}+\boldsymbol{\mu} \cdot \boldsymbol{H}
\end{aligned}
$$


where $\chi$ is the matrix of chirality parameter, and a symmtric one.

Substituting Eqs. (5) and (6) into Eqs. (124) and (125), respectively, and multiplying them with the transpose of modal matrix in the left, we have

$$
\begin{gathered}
\boldsymbol{D}^{*}=\boldsymbol{\Phi}^{T} \boldsymbol{\varepsilon} \boldsymbol{\Phi} \boldsymbol{E}^{*}-\boldsymbol{\Phi}^{T} \boldsymbol{\chi} \boldsymbol{\Psi} \nabla_{t} \boldsymbol{H}^{*} \\
\boldsymbol{B}^{*}=\boldsymbol{\Psi}^{T} \chi \boldsymbol{\Phi} \nabla_{t} \boldsymbol{E}^{*}+\boldsymbol{\Psi}^{T} \boldsymbol{\mu} \boldsymbol{\Psi} \boldsymbol{H}^{*}
\end{gathered}
$$

Let $\Gamma=\Psi^{\mathrm{T}} \boldsymbol{\chi \Phi}$, that is a coupled chiral matrix, and using Eq. (4), we have

$$
\begin{aligned}
& \boldsymbol{D}^{*}=\boldsymbol{\Lambda} \boldsymbol{E}^{*}-\boldsymbol{\Gamma}^{T} \nabla_{t} \boldsymbol{H}^{*} \\
& \boldsymbol{B}^{*}=\boldsymbol{\Gamma}^{T} \nabla_{t} \boldsymbol{E}^{*}+\boldsymbol{\Pi} \boldsymbol{H}^{*}
\end{aligned}
$$

For most chiral, $\Gamma=\operatorname{diag}\left[\varsigma_{1}, \varsigma_{2}, \varsigma_{3}\right]$. Then we have

$$
\begin{aligned}
& D_{i}^{*}=\lambda_{i} E_{i}^{*}-\varsigma_{i} \nabla_{t} H_{i}^{*} \\
& B_{i}^{*}=\varsigma_{i} \nabla_{t} E_{i}^{*}+\gamma_{i} H_{i}^{*}
\end{aligned}
$$

Eqs.(130) and (131) are just the modal constitutive equations for anisotropic chiral media.

\subsection{Eigen equations of electromagnetic waves in chiral media}

Substituting Eqs. (130) and (131) into Eqs. (23) and (24), respectively, we have

$$
\begin{gathered}
\left\{\Delta_{i}^{*}\right\} E_{i}^{*}=-\nabla_{t}\left\{\varphi_{i}\right\}\left(\varsigma_{i} \nabla_{t} E_{i}^{*}+\gamma_{i} H_{i}^{*}\right) \quad i=1,2,3 \\
\left\{\Delta_{i}^{*}\right\} H_{i}^{*}=\nabla_{t}\left\{\phi_{i}\right\}\left(\lambda_{i} E_{i}^{*}-\varsigma_{i} \nabla_{t} H_{i}^{*}\right) \quad i=1,2,3
\end{gathered}
$$

From them, we can get

$$
\begin{array}{cc}
\square_{i}^{*} E_{i}^{*}+\xi_{i} \varsigma_{i}^{2} \nabla_{t t t t} E_{i}^{*}+\left(2 \varsigma_{i} \partial_{i}^{*}+\xi_{i} \lambda_{i} \gamma_{i}\right) \nabla_{t t} E_{i}^{*}=0 & i=1,2,3 \\
\square_{i}^{*} H_{i}^{*}+\xi_{i} \varsigma_{i}^{2} \nabla_{t t t t} H_{i}^{*}+\left(2 \varsigma_{i} \partial_{i}^{*}+\xi_{i} \lambda_{i} \gamma_{i}\right) \nabla_{t t} H_{i}^{*}=0 & i=1,2,3
\end{array}
$$

where $\xi_{i}=\left\{\phi_{i}\right\}^{T} \cdot\left\{\varphi_{i}\right\}=1, \partial_{i}=\left\{\Delta_{i}^{*}\right\}^{T} \cdot\left\{\varphi_{i}\right\}$. Eqs.(134) and (135) are just equations of electric field and magnetic field for chiral media.

\subsection{Applications}

In this section, we discuss the propagation laws of electromagnetic waves in an isotropic chiral medium. The material tensors in Eqs.(124) and (125) are represented by the following matrices

$$
\boldsymbol{\varepsilon}=\left[\begin{array}{lll}
\varepsilon_{11} & 0 & 0 \\
0 & \varepsilon_{11} & 0 \\
0 & 0 & \varepsilon_{11}
\end{array}\right], \boldsymbol{\mu}=\left[\begin{array}{lll}
\mu_{11} & 0 & 0 \\
0 & \mu_{11} & 0 \\
0 & 0 & \mu_{11}
\end{array}\right], \boldsymbol{\chi}=\left[\begin{array}{lll}
\chi_{11} & 0 & 0 \\
0 & \chi_{11} & 0 \\
0 & 0 & \chi_{11}
\end{array}\right]
$$


The eigen values and eigen vectors of those matrix are the following

$$
\begin{gathered}
\boldsymbol{\Lambda}=\operatorname{diag}\left[\varepsilon_{11}, \varepsilon_{11}, \varepsilon_{11}\right], \boldsymbol{\Pi}=\operatorname{diag}\left[\mu_{11}, \mu_{11}, \mu_{11}\right], \boldsymbol{\Gamma}=\operatorname{diag}\left[\chi_{11}, \chi_{11}, \chi_{11}\right] \\
\boldsymbol{\Phi}=\boldsymbol{\Psi}=\left[\begin{array}{lll}
1 & 0 & 0 \\
0 & 1 & 0 \\
0 & 0 & 1
\end{array}\right]
\end{gathered}
$$

We can see from the above equations that there is only one eigen-space in isotropic medium, which is a triple-degenerate one, and the space structure is the following

$$
\boldsymbol{W}_{\text {mag }}=W_{1}^{(3)}\left[\boldsymbol{\phi}_{1}, \boldsymbol{\phi}_{2}, \boldsymbol{\phi}_{3}\right], \boldsymbol{W}_{\text {ele }}=W_{1}^{(3)}\left[\boldsymbol{\varphi}_{1}, \boldsymbol{\varphi}_{2}, \boldsymbol{\varphi}_{3}\right]
$$

where, $\phi_{1}^{*}=\frac{\sqrt{3}}{3}\{1,1,1\}^{T}, \varphi_{1}^{*}=\frac{\sqrt{3}}{3}\{1,1,1\}^{T}, \xi_{1}=1$.

Then the eigen-qualities and eigen-operators of isotropic chiral medium are respectively shown as follows

$$
\begin{gathered}
E_{1}^{*}=\frac{\sqrt{3}}{3}\left(E_{1}+E_{2}+E_{3}\right), \quad H_{1}^{*}=\frac{\sqrt{3}}{3}\left(H_{1}+H_{2}+H_{3}\right) \\
\square_{1}^{*}=\frac{1}{3}\left[-\left(\partial_{x}^{2}+\partial_{y}^{2}+\partial_{z}^{2}\right)\right], \quad \partial_{1}^{*}=\frac{\sqrt{3}}{3}\left(\partial_{x}+\partial_{y}+\partial_{z}\right)
\end{gathered}
$$

So, the equations of electromagnetic wave in isotropic chiral medium become

$$
\begin{aligned}
& \left(\partial_{x}^{2}+\partial_{y}^{2}+\partial_{z}^{2}\right) E_{1}^{*}=\chi_{11}^{2} \nabla_{t t t t} E_{1}^{*}+\left[2 \sqrt{3} \chi_{11}\left(\partial_{x}+\partial_{y}+\partial_{z}\right)+\frac{1}{c^{2}}\right] \nabla_{t t} E_{1}^{*} \\
& \left(\partial_{x}^{2}+\partial_{y}^{2}+\partial_{z}^{2}\right) H_{1}^{*}=\chi_{11}^{2} \nabla_{t t t t} H_{1}^{*}+\left[2 \sqrt{3} \chi_{11}\left(\partial_{x}+\partial_{y}+\partial_{z}\right)+\frac{1}{c^{2}}\right] \nabla_{t t} H_{1}^{*}
\end{aligned}
$$

where, $c$ is the velocity of electromagnetic wave

$$
c=\frac{1}{\sqrt{\mu_{11} \varepsilon_{11}}}
$$

Now, we discuss the propagation laws of a plane electromagnetic wave in x-axis. In this time, Eq.(142) becomes

$$
\frac{\partial}{\partial x^{2}} E_{1}=\chi_{11}^{2} \nabla_{t t t t} E_{1}+\left[2 \sqrt{3} \chi_{11} \frac{\partial}{\partial x}+\frac{1}{c^{2}}\right] \nabla_{t t} E_{1}
$$

Let

$$
E_{1}=A \exp [i(k x-\omega t)]
$$


Substituting the above into Eq.(145), we have

$$
k^{2}=\left(\frac{1}{c^{2}}+i 2 \sqrt{3} \chi_{11} k\right) \omega^{2}-\chi_{11}^{2} \omega^{4}
$$

or

$$
k^{2}-i 2 \sqrt{3} \chi_{11} \omega^{2} k+\left(\chi_{11}^{2} \omega^{2}-\mu_{11} \varepsilon_{11}\right) \omega^{2}=0
$$

1. when $\omega^{2}-\frac{\mu_{11} \varepsilon_{11}}{\chi_{11}^{2}}<0$

By Eq.(148), we have

$$
\begin{aligned}
& k_{1}=k_{1}^{\prime}+i k_{1}^{\prime \prime}=i \chi_{11} \omega^{2}[\sqrt{3}+(x+i y)] \\
& k_{2}=k_{2}^{\prime}+i k_{2}^{\prime \prime}=i \chi_{11} \omega^{2}[\sqrt{3}-(x+i y)]
\end{aligned}
$$

From them, we can get

$$
\begin{aligned}
& k_{1}^{\prime}=-\chi_{11} \omega^{2} y, k_{1}^{\prime \prime}=(\sqrt{3}+x) \chi_{11} \omega^{2} \\
& k_{2}^{\prime}=\chi_{11} \omega^{2} y, k_{2}^{\prime \prime}=(\sqrt{3}-x) \chi_{11} \omega^{2}
\end{aligned}
$$

where, $x=\frac{\sqrt{2}}{2} \sqrt{3+\left[9+\frac{\left(\frac{\mu_{11} \varepsilon_{11}}{\chi_{11}^{2}}-\omega^{2}\right)^{2}}{\omega^{4}}\right]^{\frac{1}{2}}}, y=\frac{\sqrt{2}}{2} \sqrt{-3+\left[9+\frac{\left(\frac{\mu_{11} \varepsilon_{11}}{\chi_{11}^{2}}-\omega^{2}\right)^{2}}{\omega^{4}}\right]^{\frac{1}{2}}}$.

Then, the solution of electromagnetic waves is the following

$$
E_{1}=A_{1} e^{-k_{1}^{\prime} x} \cdot e^{i\left(k_{1}^{\prime} x-\omega t\right)}+A_{2} e^{-k_{2}^{\prime \prime} x} \cdot e^{i\left(k_{2}^{\prime} x-\omega t\right)}
$$

It is composed of two attenuated sub-waves.

2. when $\omega^{2}-\frac{\mu_{11} \varepsilon_{11}}{\chi_{11}^{2}}>0$

By Eq.(148), we have

$$
\begin{aligned}
& k_{1}=k_{1}^{\prime}+i k_{1}^{\prime \prime}=i \chi_{11} \omega^{2}\left[\sqrt{3}+\sqrt{3+\frac{1}{\omega^{2}}\left(\omega^{2}-\frac{\mu_{11} \varepsilon_{11}}{\chi_{11}^{2}}\right)}\right] \\
& k_{2}=k_{2}^{\prime}+i k_{2}^{\prime \prime}=i \chi_{11} \omega^{2}\left[\sqrt{3}-\sqrt{3+\frac{1}{\omega^{2}}\left(\omega^{2}-\frac{\mu_{11} \varepsilon_{11}}{\chi_{11}^{2}}\right)}\right]
\end{aligned}
$$


where

$$
\begin{aligned}
& k_{1}^{\prime}=0, k_{1}^{\prime \prime}=\chi_{11} \omega^{2}\left[\sqrt{3}+\sqrt{3+\frac{1}{\omega^{2}}\left(\omega^{2}-\frac{\mu_{11} \varepsilon_{11}}{\chi_{11}^{2}}\right)}\right] \\
& k_{2}^{\prime}=-\chi_{11} \omega^{2}\left[\sqrt{3+\frac{1}{\omega^{2}}\left(\omega^{2}-\frac{\mu_{11} \varepsilon_{11}}{\chi_{11}^{2}}\right)}-\sqrt{3}\right], k_{2}^{\prime \prime}=0
\end{aligned}
$$

Then, the solution of electromagnetic waves is the following

$$
E_{1}=A_{1} e^{-k_{1}^{\prime x} x} e^{-i \omega t}+A_{2} e^{i\left(k_{2}^{\prime} x-\omega t\right)}
$$

It is seen that there only exists an electromagnetic sub-wave in opposite direction.

3. when $\omega^{2}-\frac{\mu_{11} \varepsilon_{11}}{\chi_{11}^{2}}=0$

By Eq.(148), we have

$$
k=k^{\prime}+i k^{\prime \prime}=i 2 \sqrt{3} \chi_{11} \omega^{2}
$$

where

$$
k^{\prime}=0, k^{\prime \prime}=2 \sqrt{3} \chi_{11} \omega^{2}
$$

Then, the solution of electromagnetic waves is the following

$$
E=A e^{-k^{\prime \prime} x} \cdot e^{-i \omega t}
$$

No electromagnetic sub-waves exist now.

\section{Conclusion}

In this chapter, we construct the standard spaces under the physical presentation by solving the eigen-value problem of the matrixes of dielectric permittivity and magnetic permeability, in which we get the eigen dielectric permittivity and eigen magnetic permeability, and the corresponding eigen vectors. The former are coordinate-independent and the latter are coordinate-dependent. Because the eigen vectors show the principal directions of electromagnetic media, they can be used as the standard spaces. Based on the spaces, we get the modal equations of electromagnetic waves for anisotropic media, bianisotropic media, dispersive medium and chiral medium, respectively, by converting the classical Maxwell's vector equation to the eigen Maxwell's scalar equation, each of which shows the existence of an electromagnetic sub-wave, and its propagation velocity, propagation direction, polarization direction and space pattern are completely determined in the equations. Several novel results are obtained for anisotropic media. For example, there is only one kind of electromagnetic wave in isotropic crystal, which is identical with the classical result; there are two kinds of electromagnetic waves in uniaxial crystal; three kinds of electromagnetic waves in biaxial crystal and three kinds of distorted 
electromagnetic waves in monoclinic crystal. Also for bi-anisotropic media, there exist two electromagnetic waves in Dzyaloshinskii's bi-anisotropic media, and the electromagnetic waves in bi-anisotropic medium will go faster duo to the bi-coupling between electric field and magnetic one. For isotropic dispersive medium, the electromagnetic wave is an attenuated sub-waves. And for chiral medium, there exist different propagating states of electromagnetivc waves in different frequency band, for example, in low frequency band, the electromagnetic waves are composed of two attenuated sub-waves, in high frequency band, there only exists an electromagnetic sub-wave in opposite direction, and in the critical point, no electromagnetic can propagate. All of these new theoretical results need to be proved by experiments in the future.

\section{Appendix A: Proof of the eigenmode of electromagnetic operator matrix}

The Maxwell's equation of anisotropic dielectrics is the following

$$
[\square]\{H\}=-\nabla_{t}^{2} \mu_{0}[\varepsilon]\{H\}
$$

Using the representation transform relationship Eq. (6), we have

$$
[\square][\Phi]\left\{H^{*}\right\}=-\nabla_{t}^{2} \mu_{0}[\varepsilon][\Phi]\left\{H^{*}\right\}
$$

Substituting the spectral decomposition matrix of dielectric permittivity Eq. (4) into above, we have

$$
[\square][\Phi]\left\{H^{*}\right\}=-\nabla_{t}^{2} \mu_{0}[\Phi][\Lambda]\left\{H^{*}\right\}
$$

Comparing the both sides of above equation, we can get

$$
[\square][\Phi]=-\nabla_{t}^{2} \mu_{0}[\Phi][\Lambda]
$$

Multiplying the both sides of above with the transpose of modal matrix in the left, we have

$$
[\Phi]^{\mathrm{T}}[\square][\Phi]=-\nabla_{t}^{2} \mu_{0}[\Lambda]
$$

It is seen that the right side above is a diagonal matrix, which shows that the electromagnetic operators matrix can also be spectrally decomposed in standard spaces, then we get

$$
\left[\square^{*}\right]=-\nabla_{t}^{2} \mu_{0}[\Lambda]
$$

Rewriting above in the form of scalar, we have

$$
\square_{i}^{*}=-\nabla_{t}^{2} \mu_{0} \lambda_{i}
$$

\section{Appendix B: Spectrally decomposition of lossy matrix}

The Helmholtz's free energy of electromagnetic system with lossy property is the following 


$$
\psi=\psi\left(\boldsymbol{D}, \boldsymbol{B}, \boldsymbol{D}^{d}\right)=\frac{1}{2}\left(\boldsymbol{D}-\boldsymbol{D}^{d}\right) \boldsymbol{\varepsilon}^{-1}\left(\boldsymbol{D}-\boldsymbol{D}^{d}\right)+\frac{1}{2} \boldsymbol{B} \boldsymbol{\mu}^{-1} \boldsymbol{B}
$$

Differentiating the above with lossy variable, and using Eq.(1), we have

$$
\boldsymbol{R}=-\frac{\partial \psi}{\partial \boldsymbol{D}^{d}}=\left(\boldsymbol{D}-\boldsymbol{D}^{d}\right) \boldsymbol{\varepsilon}^{-1}=\boldsymbol{E}
$$

According to the Onsager's principle, for the process of closing to equilibrium, the rate of lossy variable is proportion to the driving force, that is

$$
\frac{\partial \psi}{\partial D_{i}^{d}}+\beta_{i j} \frac{d D_{j}^{d}}{d t}=0
$$

where, $\beta_{i j}=\beta_{j i}$ is the general friction coefficient. Rewritting the above in matrix form, we have

$$
\{R\}=[B]\left\{\dot{D}^{d}\right\}
$$

Projecting the lossy electric displacement vector $\boldsymbol{D}$ into the standard spaces of the physical presentation, we get

$$
\left\{D^{d}\right\}=a\{\varphi\}
$$

Using Eq.(B2), we have

$$
\{R\}=\omega \dot{a}\{\varphi\}
$$

Substituting Eqs. (B5) and (B6) into Eq. (B4), the condition of non-zero solution to $\alpha$ is the following

$$
([B]-\omega[I])\{\varphi\}=0
$$

It is seen that the general friction coefficient matrix can also be spectrally decomposed in standard spaces, so we have

$$
[B]=[\Phi][\Omega][\Phi]^{T}
$$

Comparing Eq. (B4) with Eq. (74), it is known that we can also spectrally decompose the lossy matrix in standard spaces

$$
[\sigma]=[\Phi]^{T}[\Gamma][\Phi]
$$

where, $\boldsymbol{\Gamma}=\boldsymbol{\Omega}^{-1}=\operatorname{diag}\left[\eta_{1}, \eta_{2}, \eta_{3}\right]$.

\section{References}

[1] Agyei, A. \& Birman, J. (1990). On the linear magnetoelectric effect. Journal of Physics: Condensed Matter, Vol. 2, No. 13, (April 1990), pp. 3007-3020, ISSN 0953-8984 
[2] Astrov, D. (1960). The magnetoelectric effect in antiferromagnetics. Soviet Physics, Journal of Experimental and Theoretical Physics, Vol. 38, No. 11, (May 1960), pp. 984-985, ISSN 0038-5646

[3] Athanasiadis, C. \& Giotopoulos, S. (2003). The Atkinson-Wilcox expansion theorem for electromagnetic chiral waves. Applied Mathematics Letters, Vol. 16, No. 5, (July 2003), pp. 675-681, ISSN 0893-9659

[4] Athanasiadis, C.; Roach, G. \& Stratis, I. (2003). A time domain analysis of wave motions in chiral materials. Mathematische Nachrichten, Vol. 250, No. 1, (February 2003), pp. 3-16, ISSN 1522-2616

[5] Benveniste, Y. (1995). Magnetoelectric effect in fibrous composites with piezoelectric and piezomagnetic phases. Physical Review B, Vol. 51, No. 9, (September 1995), pp. 424427, ISSN 1098-0121

[6] Bracke, L. \& Van Vliet, M. (1981). A broadband magneto-electric transducer using a composite material. International Journal of Electronics, Vol. 51, No. 2, (February 1981), pp. 255-262, ISSN 0020-7217

[7] Bui, M.; Stuchly, S. \& Costache, G. (1991). Propagation of transients in dispersive dielectric media. IEEE Transactions on Microwave Theory and Techniques, Vol. 39, No. 7, (July 1991) , pp. 1165-1171, ISSN 0018-9480

[8] Cohen, G. (2002). Higher-order numerical methods for transient wave equations, Springer, ISBN 3-540-41598-x, Berlin

[9] Dzyaloshinskii, I. (1959). On the magneto-electrical effect in antiferromagnets. Soviet Physics, Journal of Experimental and Theoretical Physics, Vol. 10, No. 3, (April 1959), pp. 628-669, ISSN 0038-5646

[10]Dvorak, S. \& Dudley, D. (1995). Propagation of ultra-wide-band electromagnetic pulses through dispersive media. IEEE Transactions on Electromagnetic Compatibility, Vol. 37, No. 1, (February 1995), pp. 192-200, ISSN 0018-9375

[11] Gandhi, O. \& Furse, C. (1997). Currents induced in the human body for exposure to ultrawideband electromagnetic pulses. IEEE Transactions on Electromagnetic Compatibility, Vol. 39, No. 2, (February 1997), pp. 174-180, ISSN 0018-9375

[12] Guo, S. (2009). An eigen theory of electromagnetic waves based on the standard spaces. International Journal of Engineering Science, Vol. 47, No. 3, (March 2009), pp. 405-412, ISSN 0020-7225

[13] Guo, S. (2009). An Eigen theory of Static Electromagnetic Field for Anisotropic Media. Applied Mathematics and Mechanics, Vol. 30, No. 5, (May 2009), pp. 643-652, ISSN 0253-4827

[14] Guo, S. (2010). A second-order eigen theory for static electromagnetic fields. Journalof Modern Physics, Vol. 1, No. 2, (June 2010), pp. 100-107, ISSN 2153-1196

[15] Guo, S. (2010). An eigen theory of electro-magnetic acoustic waves in magnetoelectroelastic media. Acta Mechanica, Vol. 211, No. 2, (April 2010), pp. 173180, ISSN 0001-5970

[16] Guo, S. (2010). An fully dynamical theory of piezoelectromagnetic waves. Acta Mech., Vol. 215, No. 3, (December 2010), pp. 335-344, ISSN 0001-5970

[17] Haba, Z. (2004). Green functions and propagation of waves in strongly inhomogeneous media. Journal of Physis A: Mathematical and General, Vol. 37, No. 9, (September 2004), pp. 9295-9302, ISSN 0305-4470 
[18] Harshe, G.; Dougherty, J. \& Newnham, R. (1993). Theoretical modelling of multilayer magnetoelectric composites. International Journal of Applied Electromagnetic Matter, Vol. 4, No. 2, (February 1993), pp. 145-159, ISSN 1383-5416

[19] Jaggard, D.; Mickelson, A. \& Papas, C. (1979). On electromagnetic waves in chiral media. Applied Physics A: Materials Science and Processing, Vol. 18, No. 2, (February 1979), pp. 211-216, ISSN 0947-8396

[20] Jin, J,; Zunoubi, M.; Donepudi, K. \& Chew, W. (1999). Frequency-domain and timedomain finite element solution of Maxwell's equations using spectral Lanczos decomposition method. Computer Methods in Applied Mechanics and Engineering, Vol. 169, No. 2, (February 1999), pp. 279-296, ISSN 0045-7825

[21] Kong, J. (1986). Electromagnetic wave theory, John Wiley \& Sons, ISBN 978-0966814392, New York

[22] Lakhtakia, A. (1994). Beltrami fields in chiral media. World Scientific, ISBN 9810214030 , Singapore

[23] Lakhtakia, A.; Varadan, V. K. \& Varadan, V. V. (1989). Time-harmonic electromagnetic fields in chiral media. Springer-Verlag, ISBN 3-540-51317-5, Berlin

[24 ]Landau, L. \& Lifshitz, I. (1960). Electrodynamics of Continuous Media. Pergamon Press, ISBN 0-444-86165-3, Oxford

[25] Lee, R. \& Madsen, N. (1990). A mixed finite element formulation for Maxwell's equations in the time domain. Journal Computational Physics, Vol. 88, No. 2, (March 1990), pp. 284-304, ISSN 0021-9991

[26] Lindell, I.; Sihvola, A.; Tretyakov, S. \& Viitanen, A. (1994). Electromagnetic Waves in Chiral and Bi-isotropic Media. Artech House, ISBN, Boston

[27] Lindell, I.; Sihvola, A.; Tretyakov, S. \& Viitanen, A. (1994). Electromagnetic Waves in Chiral and Bi-isotropic Media., Artech House, ISBN 1598293206 , Boston

[28] Li, J, \& Chen, Y. (2006). Analysis of a time-domain finite element method for 3D Maxwell's equations in dispersive media. Computer Methods in Applied Mechanics and Engineering, Vol. 195, No. 11, (December 2006), pp. 4220-4229, ISSN 00457825

[29] Liu, Q. \& Fan, G. (1999). Simulation of GPR in dispersive media using a frequencydependent PSTD algorithm. IEEE Transactions on Geoscience and Remote Sensing, Vol. 37, No. 5, (June 1999), pp. 2317-2324, ISSN 0196-2892

[30] Lu, T.; Zhang, P. \& Cai, W. (2004). Discontinuous Galerkin methods for dispersive and lossy Maxwell's equations and PML boundaryconditions. Journal Computational Physics, Vol. 200, No. 4, (May 2004), pp. 549-580, ISSN 0021-9991

[31] Monk, P. (1992). A comparison of three mixed methods for time-dependent Maxwell's equations. SIAM Journal of Science Statistics Computer, Vol. 13, No. 10, (December 1992), pp. 1097-1122, ISSN 0960-3174

[32] Monk, P. (2003). Finite Element methods for Maxwell's equations, Clarendon Press, ISBN 0198508883, Oxford

[33] Pradhan, T. (1993). Magnetic charge induced by electric charge in a magneto-electric medium. Europhysics Letters, Vol. 21, No. 7, (July 1993), pp. 971-973, ISSN 02955075

[34] Van Run, A.; Terrell, D. \& Scholing, J. (1974). An in situ grown eutectic magnetoelectric composite material. Journal of Materials Science, Vol. 9, No. 11, (December 1974), pp. 1710-1714, ISSN 0022-2461 
[35] Yakhno, V.; Yakhno, T. \& Kasap, M. (2006). A novel approach for modeling and simulation of electromagnetic waves in anisotropic dielectrics. Internatioal Journal of Solids and Structures, Vol. 43, No. 12, (December 2006), pp. 6261-6276, ISSN 00207683 


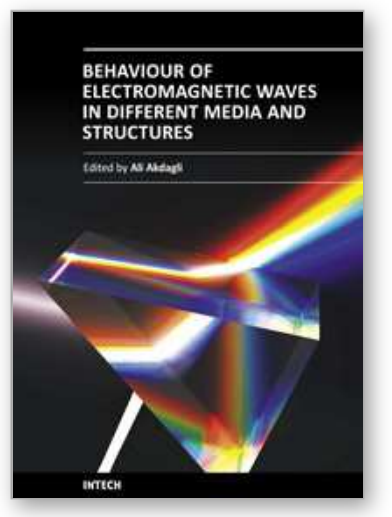

\section{Behaviour of Electromagnetic Waves in Different Media and Structures \\ Edited by Prof. Ali Akdagli}

ISBN 978-953-307-302-6

Hard cover, 440 pages

Publisher InTech

Published online 09, June, 2011

Published in print edition June, 2011

This comprehensive volume thoroughly covers wave propagation behaviors and computational techniques for electromagnetic waves in different complex media. The chapter authors describe powerful and sophisticated analytic and numerical methods to solve their specific electromagnetic problems for complex media and geometries as well. This book will be of interest to electromagnetics and microwave engineers, physicists and scientists.

\section{How to reference}

In order to correctly reference this scholarly work, feel free to copy and paste the following:

Shaohua Guo (2011). The Eigen Theory of Electromagnetic Waves in Complex Media, Behaviour of Electromagnetic Waves in Different Media and Structures, Prof. Ali Akdagli (Ed.), ISBN: 978-953-307-302-6, InTech, Available from: http://www.intechopen.com/books/behavior-of-electromagnetic-waves-in-differentmedia-and-structures/the-eigen-theory-of-electromagnetic-waves-in-complex-media1

\section{INTECH}

open science | open minds

\author{
InTech Europe \\ University Campus STeP Ri \\ Slavka Krautzeka 83/A \\ 51000 Rijeka, Croatia \\ Phone: +385 (51) 770447 \\ Fax: +385 (51) 686166 \\ www.intechopen.com
}

\author{
InTech China \\ Unit 405, Office Block, Hotel Equatorial Shanghai \\ No.65, Yan An Road (West), Shanghai, 200040, China \\ 中国上海市延安西路65号上海国际贵都大饭店办公楼 405 单元 \\ Phone: +86-21-62489820 \\ Fax: $+86-21-62489821$
}


(C) 2011 The Author(s). Licensee IntechOpen. This chapter is distributed under the terms of the Creative Commons Attribution-NonCommercialShareAlike-3.0 License, which permits use, distribution and reproduction for non-commercial purposes, provided the original is properly cited and derivative works building on this content are distributed under the same license. 